Port Acadie

Revue interdisciplinaire en études acadiennes

An Interdisciplinary Review in Acadian Studies

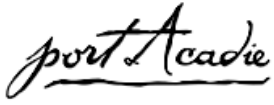

\title{
L'utilisation des TIC par les PME dans les régions rurales francophones du sud-ouest de la Nouvelle-Écosse : étude exploratoire
}

\section{Dianne Blinn et Yalla Sangaré}

Numéro 22-23, automne 2012, printemps 2013

URI : https://id.erudit.org/iderudit/1014982ar

DOI : https://doi.org/10.7202/1014982ar

Aller au sommaire du numéro

Éditeur(s)

Université Sainte-Anne

ISSN

1498-7651 (imprimé)

1916-7334 (numérique)

Découvrir la revue

Citer cet article

Blinn, D. \& Sangaré, Y. (2012). L’utilisation des TIC par les PME dans les régions rurales francophones du sud-ouest de la Nouvelle-Écosse : étude exploratoire.

Port Acadie, (22-23), 201-216. https://doi.org/10.7202/1014982ar
Résumé de l'article

À l'instar de la plupart des régions rurales du Canada atlantique, le sud-ouest de la Nouvelle-Écosse fait face à des défis économiques importants. Au-delà du déclin démographique, il y a consensus pour dire que cette région doit progressivement passer d'une économie basée sur l'exploitation des matières premières et des ressources naturelles à une économie basée sur les services et le savoir. Dans quelles mesures les francophones du sud-ouest de la Nouvelle-Écosse s'adaptent-ils aux changements structurels dans l'environnement économique? L'objet de cet article est de voir quelle utilisation les entreprises francophones de cette région font des technologies de l'information et de la communication (TIC) à partir d'un sondage réalisé auprès des dirigeants et entrepreneurs. La principale conclusion de cette étude est que les entreprises ont pris le virage des TIC, mais elles ne les utilisent pas à leur plein potentiel. 


\title{
L'utilisation des Tic par les PME dans les régions rurales francophones du sud-ouest de la Nouvelle-Écosse : étude exploratoire
}

\author{
Dianne Blinn et Yalla Sangaré \\ Université Sainte-Anne
}

\begin{abstract}
Résumé
À l'instar de la plupart des régions rurales du Canada atlantique, le sud-ouest de la Nouvelle-Écosse fait face à des défis économiques importants. Au-delà du déclin démographique, il y a consensus pour dire que cette région doit progressivement passer d'une économie basée sur l'exploitation des matières premières et des ressources naturelles à une économie basée sur les services et le savoir. Dans quelles mesures les francophones du sud-ouest de la Nouvelle-Écosse s'adaptent-ils aux changements structurels dans l'environnement économique? L'objet de cet article est de voir quelle utilisation les entreprises francophones de cette région font des technologies de l'information et de la communication (Tıc) à partir d'un sondage réalisé auprès des dirigeants et entrepreneurs. La principale conclusion de cette étude est que les entreprises ont pris le virage des Tıc, mais elles ne les utilisent pas à leur plein potentiel.
\end{abstract}

\section{Introduction ${ }^{1}$}

L'émergence d'Internet au début des années 1990 et l'essor des technologies de l'information et de la communication (Tic) ont bouleversé le fonctionnement des organisations. Pour les entreprises, l'impact se fait sentir tant en amont qu'en aval. En effet, Internet, par exemple, touche à la fois la conception, la mise en marché et le service après-vente de certains produits et services. Les Tic influent aussi sur la manière dont les gens travaillent et communiquent au sein des organisations. Jusqu'à présent, ce sont les succès spectaculaires et les échecs tout aussi retentissants de certaines sociétés - les entreprises point-com ou dotcoms - cotées en Bourse qui ont attiré l'attention ${ }^{2}$. On analyse aussi de plus en plus le rôle des médias sociaux dans la contestation de régimes autoritaires ${ }^{3}$.

1. Nous remercions le Centre d'aide en affaires et en entrepreneuriat (CAAE) et l'Université Sainte-Anne de leur appui financier et logistique à la réalisation de cette étude.

2. L'entrée récente de Linkedln à la Bourse est significative. Cette société a un chiffre d'affaires de 217 millions de dollars et a réalisé un bénéfice de 43 millions. Le prix de l'action est passé de 45 à 122 dollars dès la première séance. Ce site de réseautage social a une capitalisation boursière de plus 100 milliards de dollars. La débandade du prix de l'action du site de Facebook est tout riche d'enseignements. L'entreprise qui valait 100 milliards au moment de son entrée en bourse en mai 2012 a perdu 40 milliards en capitalisation boursière. Pour suivre la fluctuation du cours des actions des deux compagnies, voir www.nyse.com et www.nasdaq.com et le WSJ.com.

3. Il est aussi intéressant de noter que les étudiants du Québec ont fait bon usage 
La recherche néglige toutefois l'impact des Tic sur les entreprises établies dans les zones rurales. Près de 20 ans après l'émergence du commerce électronique, Internet a-t-il tenu ses promesses dans les régions éloignées? Cette étude exploratoire se propose d'aller au-delà de l'intuition et d'approfondir le sujet. Pour ce faire, nous avons réalisé un sondage auprès d'entrepreneurs et de dirigeants francophones. Notre problématique était donc la suivante : quelle utilisation les $\mathrm{PME}^{4}$ dans les régions rurales francophones et acadiennes du sud-ouest de la Nouvelle-Écosse font-elles des Tıc? En filigrane, nous avons voulu savoir si ces sociétés ont pris le virage du commerce électronique; et quand ce n'est pas le cas, quels sont les obstacles à l'essor de ce type de commerce.

La première partie de cette étude porte sur le rôle de plus en plus crucial des Tıc pour les PME et des avantages du commerce électronique ${ }^{5}$. Dans la deuxième partie, nous décrivons brièvement le sud-ouest de la Nouvelle-Écosse. Puis nous passons en revue les principaux thèmes qui reviennent dans les études sur l'utilisation des Tic dans les zones rurales. Enfin, dans la quatrième et dernière partie, nous expliquons la méthodologie utilisée et l'analyse des données proprement dite.

\section{Influence grandissante des Tic pour les entreprises}

L'objectif de cette partie est de mettre le sujet en perspective : en quoi les Tic sont-elles importantes pour les entreprises et les collectivités établies dans les zones rurales francophones et acadiennes du sud-ouest de la Nouvelle-Écosse?

Sur le plan macroéconomique, on s'entend pour dire que la richesse et la prospérité des nations ne dépendent plus de l'abondance de matières premières ou de la disponibilité d'une main-d'œuvre bon marché. Selon Porter $^{6}$, la richesse d'une nation dépend de la capacité d'innovation ${ }^{7}$ de

des médias sociaux dans le conflit du printemps 2012 qui les opposait au gouvernement libéral de la province.

4. «PME [petites et moyennes entreprises] » : il s'agit, selon Statistique Canada, des entreprises qui comptent moins de 250 employés et dont le chiffre d'affaires ne dépasse pas 50 millions de dollars canadiens.

5. "Commerce électronique »: il s'agit de l'achat, de la vente ou de l'échange de biens, de services ou d'informations en utilisant un réseau ouvert (Internet) ou fermé. La transaction se faisait traditionnellement à partir d'un ordinateur. Elle peut dorénavant se faire à partir d'un ordinateur ou de tout autre outil de communication dit « intelligent » (BlackBerry, iPad, iPhone).

6. Michael E. Porter, "The Competitive Advantage of Nations ", Boston, Harvard Business Review, mars-avril 1990, p. 73-93.

7. «Innovation » : il s'agit de la démarche par laquelle une entreprise crée de la valeur pour les parties intéressées (stakeholders). Elle peut prendre la forme d'un nouveau produit ou service, d'un nouveau procédé, d'un nouveau modèle d'affaires, etc. L'innovation doit toujours rendre la vie du consommateur plus facile. Elle 
ses entreprises et de certains secteurs industriels. On peut extrapoler et dire que ce qui est vrai pour les nations l'est aussi pour les collectivités et les régions comme le sud-ouest de la Nouvelle-Écosse. Or les Tic - et l'utilisation que l'on en fait - sont des outils qui permettent d'innover.

Dans la même optique, plusieurs études tentent de déterminer les caractéristiques des collectivités rurales innovantes. On a alors parlé de "communautés innovantes " $^{8}$. L'utilisation des Tic est l'une des caractéristiques de ces collectivités. Il est important de noter que d'autres facteurs jouent un rôle important. Dans le même ordre d'idées, les Tic permettent aux entreprises d'accroître leur compétitivité9. Or la concurrence entre les entreprises d'une même région se traduit par une plus grande prospérité pour l'ensemble de cette région.

Les Tic influent sur la manière dont l'entreprise interagit et communique avec chacune des parties intéressées (stakeholders). Elles rendent la communication plus facile et plus rapide. Prenons simplement l'exemple de la divulgation des informations financières : les rapports trimestriels sont mis en ligne et le vice-président aux finances peut commenter les résultats assez rapidement. Les Tic augmentent aussi la fréquence des communications. Et pour faire leur publicité, les entreprises disposent dorénavant de différents canaux pour communiquer avec leurs clients.

Toujours au niveau de l'entreprise, on s'entend pour dire que le Canada accuse un retard sur ses principaux concurrents en matière de productivité. Selon le Conference Board ${ }^{10}$, ceci s'explique en partie par un manque d'investissements dans la technologie. L'utilisation des Tic, la recherche-développement (R-D) et la formation de la main-d'œuvre sont les principaux moyens utilisés pour permettre aux entreprises de combler cet écart de productivité.

L'émergence du commerce électronique est l'un des événements majeurs qui se sont produits dans le monde des affaires au cours des deux dernières décennies, et ce sont les Tic qui l'ont rendue possible. Le commerce électronique connaît une croissance exponentielle et cette tendance se maintiendra au cours des prochaines années.

confère aussi un avantage à l'entreprise par rapport à la concurrence.

8. Pierre-Marcel Desjardins, "Une communauté rurale innovante et performante Quels sont les facteurs pertinents? », Université de Moncton, Institut canadien de recherche sur le développement régional, 2003, 27 p.

9. «Compétitivité d'une PME » : ce terme renvoie à la capacité qu'a la PME d'affronter la concurrence de façon efficiente et efficace. Il désigne aussi son aptitude à conquérir et à garder des parts de marché grâce à son savoir-faire et à son fairesavoir.

10. Industrie Canada, Groupe d'étude sur les politiques en matière de concurrence (consulté en ligne le 10 septembre 2010 : 〈http://www.ic.gc.ca/eic/site/cprpgepmc.nsf/fra/0o013.html). 


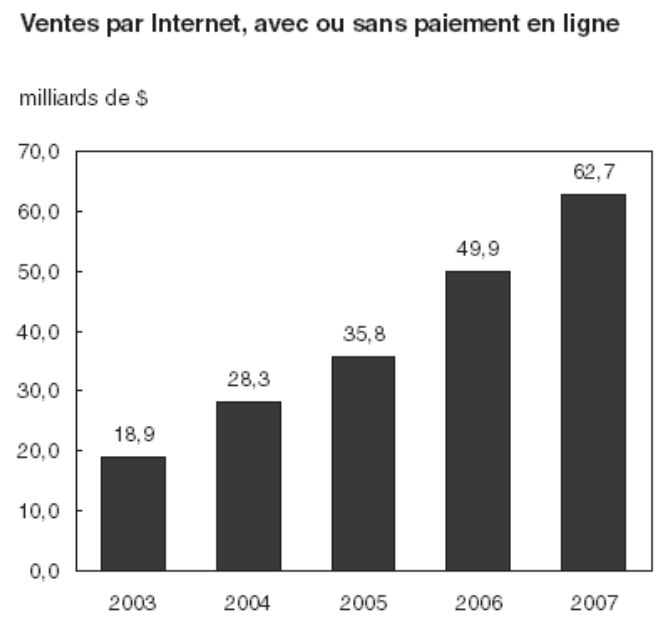

Source : Statistique Canada ${ }^{11}$

\section{Les avantages du commerce électronique pour les PME}

Le commerce électronique existe depuis deux décennies ${ }^{12}$ à peine. En quoi est-il avant-gardiste? Quels avantages offre-t-il? Quels en sont les principaux obstacles?

Le commerce électronique et les Tic changent la nature même de la concurrence $^{13}$. En effet, une PME qui vend sur le Web a dorénavant accès à un marché plus vaste. Cependant, la concurrence peut alors venir de n'importe quelle région du monde. En utilisant le modèle classique de l'analyse Moff (menaces/opportunités/forces/faiblesses), on constate que le commerce électronique crée plus d'occasions d'affaires pour les PME, mais qu'il les expose aussi à de nouvelles menaces. Ainsi, le client potentiel d'une PME est toujours "à un clic de votre concurrent ». Notre conception de l'espace et du temps change aussi : les concepts de «marché national » et de «frontières » ont tendance à s'estomper. Et les lignes de démarcation entre les industries deviennent floues.

11. Statistique Canada, Enquête surle commerce électronique et la technologie (consulté en ligne 30 mai 2011 : 〈http://www.statcan.gc.ca/daily-quotidien/080424/ dq080424a-fra.htmı).

12. Amazon, Netscape et, un peu plus tard, eBay furent les entreprises pionnières dans ce domaine.

13. Michael E. Porter, "Strategy and the Internet ", Harvard Business Review, vol. 79, $\mathrm{n}^{\circ}$ 3, mars 2001, p. 63-78. Voir aussi L'innovation et les technologies de l'information et des communications, Montréal, CefrIO, 2011, 35 p. 
Toujours sur le plan de la concurrence, le commerce électronique entraîne à la fois une « désintermédiarisation » et une « réintermédiarisation » de certains acteurs dans la chaîne de valeur. Certains intermédiaires traditionnels disparaissent. Pensons, par exemple, aux vendeurs d'ordinateurs avant l'arrivée de Dell. De nouveaux types d'intermédiaires sont apparus, le pionnier étant eBay. Depuis le début des années 2000, on assiste à l'émergence de toutes sortes d'intermédiaires (Priceline, Kijiji) qui changent notre manière de négocier. Par ailleurs, avec l'émergence des médias sociaux, le commerce interconsommateurs ( $\mathrm{C}_{2} \mathrm{C}$ ou Consumer-to-Consumer) prend plus d'ampleur, mais surtout, les clients créent de plus en plus leur propre contenu. La nature de la relation entre l'entreprise et les parties intéressées change. On passe d'un modèle classique et hiérarchisé à un modèle très hybride qui est en constante évolution. Dans ses efforts pour mieux anticiper l'avenir et demeurer concurrentiel, le gestionnaire de PME doit donc jongler avec les cubes de l'économie du savoir ${ }^{14}$ (voir les graphiques ci-après).

\section{Les cubes de l'économie du savoir}
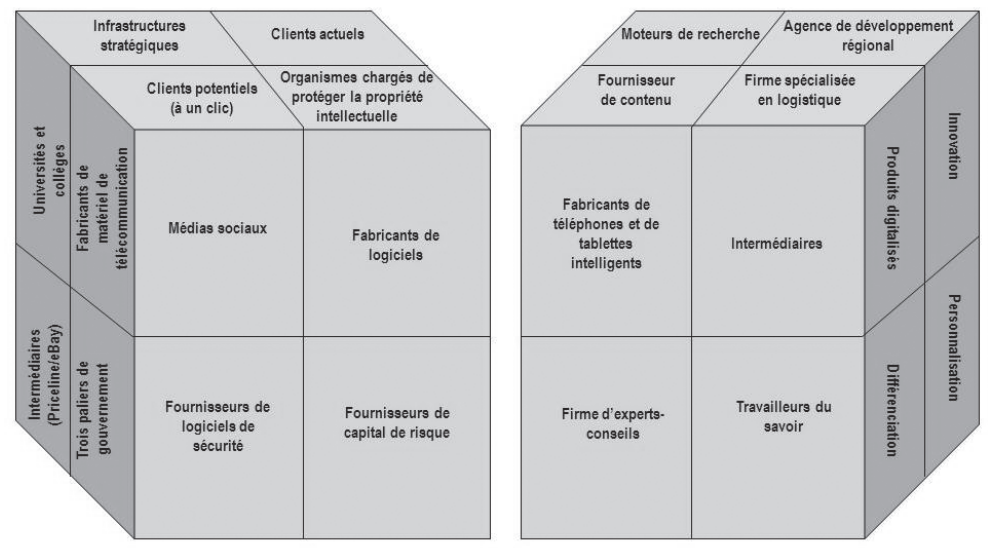

14. «Économie du savoir », « nouvelle économie » : les concepts de « nouvelle économie » et d'« économie du savoir » sont largement galvaudés. La nouvelle économie existe d'abord par opposition à l'économie industrielle. Depuis la révolution industrielle, les actifs les plus importants d'une entreprise étaient les actifs physiques (usines, camions, bâtiments, stocks, etc.). Dans une économie du savoir, l'actif le plus important est le capital humain et l'innovation est le facteur clé du succès. Dans leurs tâches quotidiennes, les employés doivent traiter beaucoup d'informations et avoir un niveau de connaissances de plus en plus élevé. 
Passage du commerce traditionnel au commerce électronique

Chaîne classique de création de valeur d'une PME

Intrants $\longrightarrow$ PME $\longrightarrow$ Client final

Chaîne de création de valeur d'une PME

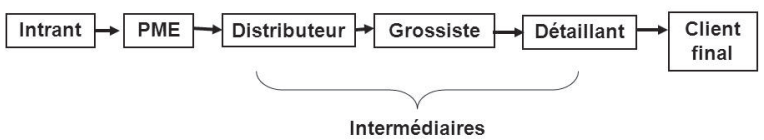

Chaîne de création de valeur simplifiée d'une PME qui vend en ligne (" Réintermédiarisation »)

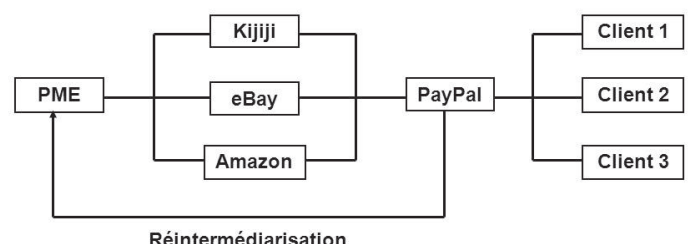

Le commerce électronique présente aussi des avantages pour le consommateur, qui a accès aux sites Web 24 heures sur 24 , sept jours sur sept. Ainsi, au lieu de se déplacer pour acheter un disque compact ou un livre, il peut dorénavant le télécharger. Le client potentiel a accès à un plus grand choix de produits et de services. Le commerce électronique est donc pratique et convivial pour l'acheteur, qui peut de plus en plus personnaliser le produit ou le service de son choix. Il est aussi facile de comparer les produits et les services sur le plan de la qualité et du prix. Le consommateur est mieux informé et jouit d'une plus grande souplesse en fonction de son pouvoir d'achat, grâce aux nouveaux modèles de tarification (enchères sur eBay, offres et enchères inversées Priceline, rabais en fonction du nombre d'acheteurs chez Groupon, etc.). En somme, le consommateur court un moins grand risque, particulièrement lorsqu'il se procure des biens dispendieux. Le commerce électronique facilite aussi la prise de décision.

Les autres avantages du commerce électronique pour les PME sont bien connus. Comme nous l'avons souligné, les entreprises peuvent rejoindre une clientèle plus large. Le commerce électronique leur permet une meilleure observation du comportement du consommateur, que l'on peut " suivre " dès qu'il arrive sur le site Web et jusqu'au moment où il le 
quitte. Les entreprises peuvent donc faire des économies substantielles sur le plan des coûts ${ }^{15}$ et elles sont en mesure de personnaliser leur offre de produits et de services. Le commerce électronique et les Tic en général permettent aussi aux entreprises de mieux gérer leur logistique. Au final, le commerce électronique peut, dans certains contextes, procurer un avantage compétitif en permettant aux entreprises de se distinguer de leurs concurrents.

L'expansion du commerce électronique fait face à un certain nombre d'obstacles ${ }^{16}$. Les coûts qu'il entraîne sont le principal frein à l'adoption du commerce électronique par les PME. En effet, ces entreprises n'ont pas toujours les moyens de s'offrir les technologies les plus chères. Le deuxième obstacle a trait à la sécurité et touche les entreprises et les clients potentiels, car ces derniers hésitent à divulguer des informations confidentielles à une PME plus ou moins connue. On remarque que les PME sont plus inquiètes pour leur sécurité que les grandes sociétés. Un des autres freins à l'expansion du commerce électronique a trait à la nondisponibilité d'une main-d'œuvre qualifiée, particulièrement dans les zones rurales. Il ne s'agit pas vraiment d'un « obstacle», mais l'émergence

\section{Avantages du commerce électronique pour les PME}

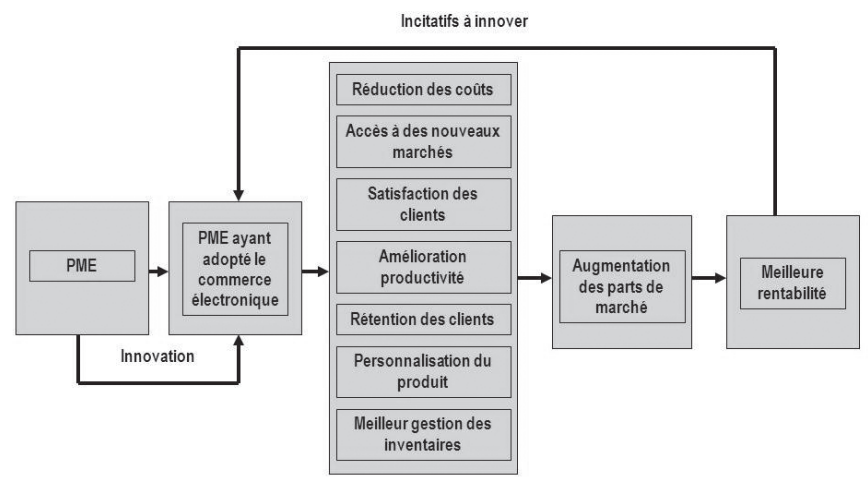

15. Daniel Zéghal et Yalla Sangaré, «E-commerce: New Challenge for Small and Medium Business », Université d'Ottawa, Telfer School of Management, document de travail, $n^{\circ} 11,2002,14 \mathrm{p}$.

16. Élaine M.-F Moreau, Sylvain Delisle, Louis Raymond et Bernard Vermot-Desroches, «Affaires électroniques et PME : naissance d'une nouvelle économie pour le développement régional », Université du Québec à Trois-Rivières, Cahier de recherche de L'INRPME, 2008, p. 6. 
des médias sociaux pose un certain nombre de défis aux entreprises qui font du commerce en ligne, car elles doivent combiner les outils de marketing traditionnels à ceux des médias sociaux.

\section{Le sud-ouest de la Nouvelle-Écosse ${ }^{17}$}

Afin de comprendre l'utilisation que les entrepreneurs du sud-ouest de la Nouvelle-Écosse font des Tic, il faut comprendre le contexte dans lequel ils évoluent. La Nouvelle-Écosse est l'une des provinces qui font partie du Canada dès la fondation du pays, en 1867. La population de cette province s'élève aujourd'hui à 940000 habitants (www.statcan.gc.ca) et augmente sensiblement d'un recensement à l'autre. Cependant, cette croissance cache de grandes disparités régionales. En effet, la population de l'agglomération d'Halifax augmente, mais le nombre d'habitants diminue partout ailleurs dans la province. L'économie de la Nouvelle-Écosse repose sur le tourisme et sur les matières premières, et les trois paliers de gouvernement sont aussi de grands employeurs.

Le sud-ouest de la Nouvelle-Écosse est composé des comtés de Digby, de Yarmouth et de Shelburne. La municipalité de Clare se trouve dans le comté de Digby et celle d'Argyle dans le comté de Yarmouth. À cet égard, certaines observations s'imposent.

Sur le plan démographique, une analyse horizontale des résultats du recensement dans ces trois comtés montre que la population diminue dans le sud-ouest de la Nouvelle-Écosse. Elle a aussi tendance à être plus âgée qu'ailleurs dans la province et au Canada. Cette situation s'explique par le départ des jeunes, qui vont chercher de meilleurs débouchés à Halifax ou dans l'Ouest canadien.

Sur le plan économique, les emplois dans cette région sont saisonniers. Par ailleurs, le taux de chômage y est beaucoup plus élevé que dans l'ensemble de la province, alors que le revenu moyen et le revenu médian des citoyens sont plus bas. Les tableaux de la répartition par secteur montrent que les secteurs primaire et secondaire emploient encore un nombre élevé d'habitants du sud-ouest. De façon plus concrète, les principales industries sont la pêche, l'industrie forestière et la transformation des produits de la pêche. Les services gouvernementaux et le tourisme constituent le secteur des services. Comme dans toutes les autres provinces canadiennes, plus de 60 p. 100 des exportations de la Nouvelle-Écosse se font à destination des États-Unis.

17. Pour un profil des régions rurales en Nouvelle-Écosse, voir Painting the Landscape of Rural Nova Scotia, Rural Communities Impacting Project (RCIP) Policy Project, 2003, 105 p., en particulier p. 7-46. 
Sur le plan sociologique, la région du sud-ouest de la Nouvelle-Écosse est peuplée de descendants des Premières Nations, d'Acadiens, d'anglophones et d'un très faible nombre d'immigrants.

En résumé, le sud-ouest de la Nouvelle-Écosse est une région dont la population vieillissante dépend largement du secteur des ressources naturelles et de l'industrie du tourisme. Cette région fait face à trois défis supplémentaires : tout d'abord, comme nous l'avons souligné, plus des deux tiers des exportations vont vers les États-Unis. Or l'économie américaine se remet très lentement de la dernière récession. Au cours des derniers trimestres, le dollar canadien a fortement augmenté par rapport à la devise américaine, ce qui pénalise les exportateurs ${ }^{18}$. L'industrie du tourisme risque aussi de subir les contrecoups de cette augmentation. Les exigences en matière de sécurité (avec l'obligation de présenter un passeport pour traverser la frontière) dissuadent certains touristes potentiels. Enfin, de tout temps, le sud-ouest a été relié à la Nouvelle-Angleterre par un traversier. Or, depuis deux ans, ce service a été aboli, ce qui a eu un impact négatif sur le comté de Yarmouth. Ajoutons à cela l'absence de transport aérien, et on peut facilement dire que la région est de plus en plus enclavée.

Cette région offre aussi des avantages et jouit d'un fort potentiel. Outre ses attraits touristiques inestimables, elle possède d'énormes ressources sur le plan des énergies renouvelables. La région exporte beaucoup de produits à l'état brut et sans beaucoup de valeur ajoutée. La transformation de ces produits par des entreprises locales peut créer plusieurs centaines d'emplois. Enfin, la région a une université et deux collèges situés dans un rayon d'environ 150 kilomètres. Or très peu de régions rurales peuvent se targuer d'avoir une telle concentration d'institutions du savoir à si courte distance. Ces établissements d'enseignement peuvent jouer le rôle de catalyseurs, voire de locomotives, pour favoriser une transition vers l'économie du savoir.

\section{La municipalité d'Argyle}

Sur le plan démographique, la population d'Argyle s'élève à 8655 habitants $^{19}$, qui occupent une superficie de 1527 kilomètres carrés. Près de la moitié de ces personnes sont francophones (46,5 p. 100, selon

18. Le dollar canadien s'est déprécié au début 2012 et depuis le début de l'été s'échange à parité avec le dollar américain. La grande inconstance du taux de change a certainement un impact négatif sur les échanges avec l'étranger. Elle a cependant le mérite de forcer les entreprises à innover et à améliorer leur productivité. Les Tic peuvent jouer un rôle important.

19. Profil communautaire 2008 - Communauté acadienne et francophone de la région d'Argyle, CDENE/RDÉE, 2008, 106 p. 
le dernier recensement). Comme dans l'ensemble du sud-ouest, l'économie de cette municipalité dépend de la pêche et du tourisme.

\section{La municipalité de Clare 20}

La municipalité de Clare s'étend sur une distance de 50 kilomètres, le long de la baie Sainte-Marie. En 2006, la population s'élevait à 8815 habitants, répartis sur une superficie de 852,6 kilomètres carrés. Selon le dernier recensement, 65,8 p. 100 ont le français comme langue maternelle.

La région du sud-ouest est enclavée. L'aéroport le plus proche, celui de Halifax, se trouve à 300 kilomètres de Clare. Le sud-ouest fait face à de grandes difficultés, mais possède aussi un potentiel énorme. On peut donc affirmer que la bonne utilisation des Tic représente un net avantage pour cette région.

\section{Les principales études dans ce secteur}

Dans les écrits portant sur l'utilisation que les PME établies dans les zones rurales francophones font des Tic, quatre thèmes principaux reviennent constamment.

La "fracture numérique ${ }^{21}$ est sans aucun doute le thème majeur. Ce concept renvoie au fait que les entreprises et les citoyens des zones rurales ont moins accès à une connexion Internet à haut débit que ceux des centres urbains. Au-delà de la connexion à Internet, cette fracture porte aussi sur l'accès des régions rurales à l'ensemble des Tic. Au Canada, cette fracture est due à la géographie des lieux. En effet, le territoire est vaste et peu peuplé. Les entreprises de télécommunication estiment donc qu'il n'est pas rentable pour elles d'offrir leurs services aux populations établies dans les zones rurales éloignées. Leur réticence est d'autant plus grande qu'en plus de la faible densité, la population de ces régions tend à diminuer. La fracture numérique ${ }^{22}$ comporte une autre dimension : les

20. Profil communautaire 2008 - Communauté acadienne et francophone de la région de Clare, CDENE/RDÉE, 2008, 105 p.

21. George Sciadas, "La fracture numérique au Canada », Ottawa, Statistique Canada, document de recherche $n^{\circ}$ 56FoogXIF, 5 p. [consulté en ligne le 30 novembre 2010 : http://publications.gc.ca/Collection/Statcan/56Fooo9X/56FooogXIF2002001. pdf]. Larry McKeown, Anthony Noce et Peter Czerny, « Facteurs associés à l'utilisation de l'Internet : une question de ruralité? », Ottawa, Statistique Canada, Bulletin d'analyse - Région rurale et petites villes du Canada, vol. 7, n³, 2007 [consulté le 22 mars 2011 : «http://www.statcan.gc.ca/pub/21-006-x/21-006-x2007003-fra. pdfı]. Danielle Galliano et Pascale Roux, «Les Inégalités spatiales dans l'usage des Tıc: le cas des firmes industrielles françaises ", Revue économique, vol. 57, $\mathrm{n}^{\circ} 6$, p. $1449-1475$.

22. "Fracture numérique ": "Le terme "fracture numérique", c'est-à-dire l'écart entre les "nantis » et les démunis de Tic, comporte de nombreux enjeux, comme 
frais de communication, qui sont plus élevés pour les entreprises dans les zones rurales. Conscients de ces obstacles, les gouvernements subventionnent la construction d'infrastructures stratégiques, comme les réseaux de fibre optique. Cette fracture lance donc un débat qui porte sur la disponibilité d'infrastructures dites «stratégiques».

Le deuxième thème porte sur la relation de cause à effet entre l'utilisation des Tic par les entreprises et le développement économique des zones rurales. Les études menées cherchent à savoir si les Tic sont uniquement des catalyseurs ou si elles sont aussi des facteurs de développement économique. Ici, les travaux de recherche vont au-delà de la situation des entreprises et s'intéressent à l'ensemble de l'utilisation qui est faite des Tic dans les zones rurales. Certains travaux sont des études de cas qui cherchent à déterminer si la présence ou l'absence d'Internet à haut débit a un impact sur le développement économique et social ${ }^{23}$.

Les recherches traitent aussi d'un autre volet important : celui des difficultés que doivent affronter les PME établies en zones rurales lorsqu'elles décident de passer au commerce électronique. La première difficulté est l'absence de main-d'œuvre qualifiée. Les PME ont aussi du mal à combiner leurs systèmes informatiques traditionnels et ceux du commerce électronique. Par ailleurs, les entrepreneurs dans les zones rurales ne voient pas de façon quantifiable l'impact qu'aurait l'adoption des Tic sur leur marge bénéficiaire. En général, dans ces régions, la formation est moins disponible et la culture organisationnelle ne favorise pas l'adoption des Tic.

Les divers facteurs qui incitent les entreprises qui se trouvent dans les zones rurales à adopter - ou non - les Tic constituent un autre des thèmes abordés par les chercheurs ${ }^{24}$. Les entreprises établies dans les zones urbaines adoptent plus rapidement les nouvelles technologies et les utilisent aussi de façon plus intense. Qu'en est-il des PME en zone rurale? II convient ici de faire une distinction entre les facteurs internes et externes. Ainsi, l'environnement concurrentiel, les relations avec les

l'infrastructure et l'accès aux Tic, leur utilisation, leurs contraintes ainsi que le rôle fondamental de la culture et des habiletés relatives aux Tic pour évoluer vers une société d'information. » (Georges Sciadas, op. cit., p. 1)

23. Sid-Ahmed Selouani et Habib Hamam, "Social Impact of Broadband Internet: A Case study in the Shippagan Area, a Rural Zone in Atlantic Canada », Journal of Information, Technology and Organizations, vol. 2, 2007, p. 80-94.

24. S. Shong et G. Pervan, «Factors influencing the extent of deployment of electronic commerce for small and medium-sized enterprises ", Journal of Electronic Commerce in Organizations, vol. 5, n 1, 2007, p. 1-29; Elaine Moreau, "Affaires électroniques pour le développement des PME : bilan et perspectives ", dans les actes de CIFEPME, 2010, 16 p. [consulté en ligne le 30 mai 2012: «http://Web.hec. ca/airepme/images/File/2010/MOREAU-CIFEPME2010.pdf_] 
fournisseurs, la disponibilité d'infrastructures et la proximité d'une agglomération peuvent inciter certaines sociétés à adopter les Tic. Il y a aussi des facteurs internes qui jouent et qui peuvent aller du type de produit jusqu'au mode d'interaction avec les principaux intervenants internes. Nous y reviendrons plus loin, mais disons qu'il n'est pas rare d'entendre des entrepreneurs des zones rurales dire que leurs produits et services ne se prêtent pas bien au commerce électronique.

Un des thèmes centraux qui vont au-delà des zones rurales concerne le lien entre l'utilisation des Tic et l'innovation. Le sujet est abordé sous différents angles. Cependant, on veut savoir comment les Tic permettent l'émergence de nouveaux modèles d'affaires, de nouveaux produits ou services, de nouveaux procédés, etc. On cherche à établir la relation de cause à effet entre les Tic et l'innovation, et entre cette dernière et la prospérité d'une région, voire d'un pays ${ }^{25}$.

Le quatrième et dernier thème traite de l'impact réel et mesurable du commerce électronique sur "l'amélioration de la performance des $P M E »^{26}$. Le commerce électronique procure-t-il un avantage concurrentiel à l'entreprise? Selon certaines études, celui-ci peut donner un avantage concurrentiel à l'entreprise, mais à certaines conditions, qui varient en fonction du contexte.

L'utilisation et surtout l'adoption des Tic se font dans un environnement particulier, qui a un impact énorme sur les pratiques des entreprises et sur leur utilisation de ces technologies. Les nombreuses études sur le sujet ont montré que la Silicon Valley devait ses succès à un certain nombre de facteurs : la présence dans une même région d'une masse critique d'entreprises (on parle de « grappe ») œuvrant dans le même secteur, la disponibilité du capital de risque, la présence d'institutions du savoir et l'existence d'une culture qui avait une grande tolérance vis-à-vis du risque et de l'échec ${ }^{27}$. Dans le même ordre d'idées, certaines études essaient de déterminer les traits et les caractéristiques des entreprises qui innovent dans les régions rurales, mais on sait déjà que la disponibilité d’infrastructures stratégiques et l'utilisation des Tic en font partie.

25. Soumitra Dutta et Irene Mia (dir.), The Global Information Technology Report 20102011, Genève, INSEAD, World Economic Forum, 2011, 435 p. [consulté en ligne le 20 octobre 2011]

26. Moez Bellaaj, "Effet modérateur des capacités complémentaires dans le e-commerce : application au contexte des PME », Revue internationale PME, vol. 22, $\mathrm{n}^{\circ}$ 2, 2009, p. 9-40.

27. Pour comprendre la culture de «la vallée », voir entre autres : Michael Lewis, The New New Thing: A Silicon Valley Story, New York, Norton \& Company, 1999, 256 p.; Anna Lee Saxenian, "Silicon valley : les secrets d'une réussite ", Sciences humaines, hors-série n²9, 2000 [consulté en ligne le 6 juin 2012 : «http://www. scienceshumaines.com/silicon-valley-les-secrets-d-une-reussite_fr_12105.html〉]. 


\section{Analyse des données recueillies}

\section{Méthodologie}

Certains éclaircissements s'imposent quant à la méthodologie que nous avons utilisée. Tout d'abord, notre étude est exploratoire. En effet, il n'existe aucune recherche sur le sujet et sur la région du sud-ouest de la Nouvelle-Écosse. Notre population de départ est constituée de l'ensemble des entreprises inscrites dans les annuaires du Conseil de développement économique de la Nouvelle-Écosse (CDÉNÉ). Nous avons sélectionné de façon aléatoire 110 entreprises des municipalités de Clare et d’Argyle. De cet échantillon, nous avons obtenu un taux de réponse de 55,45 p. 100 à notre sondage téléphonique. Les pourcentages mentionnés dans l'analyse ci-dessous ont donc trait à ceux qui ont répondu au questionnaire. Nous n'avons pas fait d'inférence. Le questionnaire qui a servi de base au sondage a été "pré-testé » et testé auprès d'universitaires et d'entrepreneurs. Nous avons circonscrit notre étude à deux municipalités : celle de Clare et celle d'Argyle. Nous avons aussi discuté des résultats obtenus avec des membres de la chambre de commerce locale, afin de voir s'ils reflètent la réalité observée.

\section{Analyse}

Notre questionnaire comportait 40 questions et cinq sections. La première et la dernière section ont servi essentiellement à recueillir des informations sur les entreprises. Une section portait sur l'utilisation des Tic en général, une autre sur l'utilisation d'Internet et une autre encore sur le commerce électronique.

La première constatation qui s'impose est la suivante : les entreprises francophones du sud-ouest de la Nouvelle-Écosse ont pris le virage des Tic. En effet, neuf entreprises sur 10 utilisent des ordinateurs et sont branchées à Internet. Elles ont accès à Internet à haut débit dans les mêmes proportions. Elles utilisent les ordinateurs d'abord pour la comptabilité (91 p. 100), pour naviguer sur le Web (85 p. 100) et pour le traitement de texte (70 p. 100). Nos chiffres montrent cependant que moins de 50 p. 100 d'entre elles utilisent une quelconque base de données.

En ce qui a trait au commerce électronique, moins de 50 p. 100 des entreprises achètent ou vendent en ligne. Les chiffres exacts sont les suivants : 42 p. 100 font des achats en ligne et 48 p. 100 font des ventes en ligne. Un peu plus de la moitié des entreprises ont un site Web (52 p. 100). Les répondants qui n'ont pas de site donnent différentes raisons, la principale étant le coût. Par ailleurs, certains estiment qu'un site ne leur serait d'aucune utilité. 
Les entreprises qui ont un site Web sont satisfaites de cet outil dans une proportion de 75 p. 100. Elles considèrent, à 63 p. 100, que les ventes à partir de leur site leur ont permis d'augmenter leur chiffre d'affaires et de rejoindre une clientèle qui, autrement, n'était pas atteignable. Enfin, à peine 25 p. 100 des entreprises utilisent des outils de mesure de performance qui leur permettent de connaître le nombre de visiteurs, leur provenance et le temps qu'ils passent sur leur site. Un chiffre plutôt surprenant : lorsqu'on leur demande si cela les intéresserait de suivre une formation si on en mettait une à leur disposition, 78 p. 100 des chefs d'entreprise répondent que non. Les Tic ne semblent pas permettre d'améliorer la productivité ${ }^{28}$ (à peine 6 p. 100 répondent oui à cette question) ou de réaliser des économies.

À l'issue de cette analyse, trois observations s'imposent. D'abord, les entreprises des municipalités d'Argyle et de Clare utilisent les technologies de l'information dans le cadre de leurs activités normales : comptabilité, traitement de texte, recherches sur le Web. Ensuite, les entreprises francophones du sud-ouest de la Nouvelle-Écosse n'ont pas pris le virage du commerce électronique. Ceci est particulièrement vrai pour ce qui est de la vente en ligne. Enfin, même les entreprises qui ont pris le virage électronique n'exploitent pas tout le potentiel qu'offrent les Tic. Ceci est particulièrement vrai au chapitre des outils de mesure de performance (Google Metrics) et de l'utilisation des outils disponibles pour faire du marketing en ligne. Les entrepreneurs du sud-ouest n'utilisent pas assez les Tic pour innover et améliorer leur productivité.

Il faut utiliser ces résultats avec beaucoup de prudence, puisqu'il s'agit d'une première étude sur le sujet. Il faudra peut-être la refaire tous les deux ans et observer les tendances sur une décennie. La plupart des entreprises des deux régions œuvrent dans les secteurs primaire et secondaire, les produits et services se prêtant peu au commerce électronique. La plupart des propriétaires d'entreprise dans les deux municipalités sont des baby-boomers ou des membres de la génération $X$ (citoyens nés entre 1965 et 1980). Les nouvelles générations d'entrepreneurs et de dirigeants d'entreprises ont grandi avec les $\mathrm{Tic}^{29}$. Au fur et à mesure qu'ils assureront la relève, ils feront prendre le virage électronique aux entreprises de ces

28. «Productivité »: rapport entre les intrants et les extrants. Selon Statistique Canada, " [l]a croissance de la productivité est mesurée en comparant l'accroissement de la production par rapport à celui des ressources utilisées dans la production. Une valeur positive de la productivité est alors associée à une augmentation de l'efficience ». [consulté en ligne le 22 mars 2012 : 〈http://publications.gc.ca/Collection/ Statcan/15-206-XIF/15-206-XIF2006002.pdf `].

29. Don Tapscott, Grown Up Digital: How the Net Generation is Changing Your World, New York, McGraw Hill Companies, 2009, 308 p. 
deux municipalités. Enfin, le retard que le sud-ouest accuse est principalement dû au coût élevé de ces nouvelles technologies, surtout lorsque la main-d'œuvre n'est pas disponible.

\section{Conclusion}

Nous avons entrepris une étude exploratoire afin de voir quelle utilisation les entreprises font d'Internet dans les zones rurales francophones et acadiennes du sud-ouest de la Nouvelle-Écosse. Notre principale conclusion est que les entreprises des municipalités de Clare et d'Argyle utilisent les Tıc, mais pas à leur plein potentiel. Elles achètent et vendent encore de manière traditionnelle et n'ont pas pris le virage du commerce électronique. Elles n'utilisent pas encore les technologies pour innover ou améliorer leur productivité.

Cette étude comporte certaines limites et nous en sommes conscients. Nous avons fait notre échantillonnage à partir d'une population constituée de l'ensemble des entreprises inscrites dans les bottins du Conseil de développement économique de la Nouvelle-Écosse. Malgré les efforts de cet organisme, il est possible que toutes les entreprises ne s'y trouvent pas. En effet, beaucoup d'entreprises établies dans les municipalités de Clare et d'Argyle sont saisonnières, et il est probable qu'il y avait une surpondération du nombre d'entreprises qui étaient ouvertes au moment du sondage. Par ailleurs, certaines entreprises ont des propriétaires anglophones et francophones, et certaines des sociétés appartenant à des francophones se trouvent dans les régions anglophones avoisinantes. Le sondage a été effectué à la fin du deuxième semestre de 2009 et au début de 2010. Cette période correspond à la fin de la crise économique la plus grave que nous ayons connue depuis 1929. En général, dans de tels contextes, les entreprises sont prudentes dans leurs investissements. Enfin, au moment où nous avons rédigé notre questionnaire, les médias sociaux étaient encore à l'état embryonnaire. Ils jouent un rôle de plus en plus important et les PME les utilisent beaucoup. Toute étude future devra comporter des questions sur les médias sociaux, afin de tenir compte de leur importance grandissante. En effet, une PME peut ne pas avoir un site Web de la dernière génération tout en étant active sur Facebook. Il faut pouvoir comparer les données à celles des autres régions rurales francophones ou même anglophones. En dépit de ces limites, nous sommes convaincus que les éléments de notre conclusion traduisent la réalité observée quant à l'utilisation des Tıc dans le sud-ouest de la Nouvelle-Écosse.

Pour l'avenir, certaines remarques s'imposent. Beaucoup d'entreprises des municipalités de Clare et d'Argyle sont de petites entreprises, voire des micro-entreprises. La première étape consiste à les sensibiliser à 
l'importance des Tic. Il faut absolument élaborer pour elles une formation sur mesure et, surtout, les sensibiliser à l'importance et aux avantages qu'elles pourront tirer de l'adoption du commerce électronique. À ce chapitre, les chambres de commerce et surtout les institutions du savoir ont un rôle essentiel à jouer. Des possibilités s'offrent aux entreprises qui n'ont pas les moyens d'avoir un site Web interactif, car elles peuvent ouvrir des magasins virtuels sur eBay ou sur Amazon. Elles peuvent aussi se rendre sur les sites plus spécialisés, comme etsy.com ou Groupon, qui peuvent permettre aux entreprises qui n'ont pas beaucoup de moyens de prospérer.

Sur un plan plus personnel, il faut aussi être conscient du fait que les Tic peuvent permettre d'améliorer la qualité de vie des habitants des zones rurales et leur offrir des possibilités qui, autrement, n'existeraient pas, en plus d'entraîner une diversification de l'économie.

Il existe très peu d'études sur les facteurs expliquant l'utilisation des Tic par les entreprises francophones dans les maritimes. Il est impératif de mettre sur pied un système de veille technologique ${ }^{30}$. Ainsi, en utilisant, par exemple, les réseaux de développement économique et d'employabilité (RDÉE), les entrepreneurs peuvent mettre en commun les meilleures pratiques.

Enfin, les études futures devront s’intéresser au problème que pose l'adoption des Tic. Pourquoi les entrepreneurs sont-ils plus ou moins nombreux à les adopter? S'ils avaient une meilleure compréhension du commerce électronique, sans doute seraient-ils plus nombreux à l'adopter.

Bien entendu, il ne peut être question de Tıc sans parler de la disponibilité des infrastructures stratégiques, qui pose un problème majeur dans les zones rurales, compte tenu de l'usage de plus en plus répandu des appareils dits « intelligents ». Il est important que les entreprises francophones prennent le virage électronique, dans la mesure où les jeunes, qui sont aussi leurs futurs clients, sont de plus en plus nombreux à utiliser ces appareils technologiques.

30. "Veille concurrentielle : : la veille concurrentielle consiste, pour une entreprise, à surveiller ce qui se passe dans son secteur. Elle "scanne » constamment l'environnement afin d'être à l'affût des opportunités et des menaces. 